International Journal of Advanced Biological and Biomedical Research Available online at http:www.ijabbr.com

Volume 7, Issue 4 (2019) pp. 326-334

DOI: 10.33945/SAMI/IJABBR.2019.4.4

Original Article

\title{
Effect of Sub-effective dose of GABA Agonists on Attenuation of Morphine Tolerance in Rats: Behavioral and Electrophysiological Studies
}

\section{Shima Mehrabadi' ${ }^{1}$, Homa Manaheji ${ }^{2, *}$}

1Department of Physiology, Faculty of Medicine, Tehran University of Medical Sciences, Tehran, Iran

${ }^{2}$ Neurophysiology Research Center, Shahid Beheshti University of Medical Sciences, Tehran, Iran

*Corresponding Author E-mail: kumarajoy.cu@gmail.com

Received: 1 April 2019, Revised: 28 May 2019, Accepted: 30 May 2019

\begin{abstract}
GABAergic drugs can change analgesic effect of morphine. Wide dynamic range (WDR) neurons play an important role in pain transmission and may change behaviors in morphine tolerance. In this study, WDR neuron behaviors in morphine tolerant rats and rats treated with GABA agonists, were recorded to elucidate the effect of morphine and GABA agonists on WDR behavioral changes. Rats were divided to 4 groups: 1. Control, 2. Morphine tolerance (MT), 3. MT+ muscimol, 4- MT+ baclofen. To induce morphine tolerance in rats, they received morphine sulfate $10 \mathrm{mg} / \mathrm{kg}$ intraperitoneally for 8 days. In the treatment group, GABA agonists were injected on days 1, 3, 5 and 8 before injection of morphine. To confirm morphine tolerance induced, formalin test was used. Extracellular single unit recording was used to record spinal WDR neurons. Results showed that chronic administration of morphine failed to attenuate formalin pain but GABA agonists improved analgesic effect of morphine.
\end{abstract}

Key words: Morphine tolerance, GABA, WDR, Electrophysiology, Formalin test.

\section{Introduction}

Morphine is an effective drug in improving acute and chronic pain syndrome. Especially in chronic pain, it can attenuate pain, improve patient's life and increase life expectancy in people with chronic pain. (Lilius et al., 2017). Although morphine is the most efficient drug in chronic pain but chronic administration of morphine leads to the development of tolerance to the anti-nociception effect of morphine. Tolerance limits the use of opioids and therefore co-administration of other drugs with chronic administration of morphine is considered (Mansouri et al., 2015). After several decades of study on morphine tolerance, it is still not understood very well. One of the most possible causes of tolerance is alternation on neurotransmitter's level (Mehrabadi and Karimiyan, 2018). There are several reports about alternation of GABA neurotransmitter on opioid tolerance and dependence (Dobashi 
et al., 2010; Hull et al., 2013). Recently, behavioral and molecular studies showed that GABA agonists could be used in the treatment of opioid tolerance and improve the alternation of GABA level in morphine tolerance and dependence (Vorma and Katila, 2011). Systemic administration of GABA agonists attenuated withdrawal syndrome induced by naloxone (Belozertseva and Andreev, 2000). GABA has an important role in pain modulatory pathway that is activated by morphine and affects spinal cord (Lueptow et al., 2018). The main center of action of morphine is the dorsal horn of the spinal cord (Haugan et al., 2008). There is no investigation that clarifies the effect of morphine tolerance and effect of GABA agonists on electophysiological changes in spinal cord level. In the present study, subeffective dose of GABA agonists that did not induce analgesia was determined to investigate the effect of GABA on morphine analgesic in chronic administration. Then, the effect of morphine tolerance on WDR neurons behavior was investigated as one of the most important neurons which has a role in pain transmission on spinal cord. Moreover, the effect of different GABA agonists on electrophysiological changes of WDR neurons was investigated to understand the possible effect of morphine tolerance and co-administration of GABA agonists with morphine in chronic use at spinal cord level and WDR neuron's behaviors.

\section{Materials and methods}

\section{Animals}

64 male wistar rats weighing 200-250 gr were used in this study (n=8 per group). They were housed four per cage under a $12 \mathrm{~h}$ light/dark cycle in a room with controlled temperature $\left(22 \pm 1^{\circ} \mathrm{C}\right)$. Food and water were available ad libitum.

\section{Protocol}

First, to find sub effective dose of GABA agonists, three different doses of drug were used as a single dose injection $(0.5,0.75$ and $1 \mathrm{mg} / \mathrm{kg}$; i.p.) in normal rats before the formalin test. Baclofen and muscimol ( $0.5 \mathrm{mg} / \mathrm{kg}$, i.p.) had no analgesic effect on the normal rats in the formalin test, thus, these doses were selected for the current studies to evaluate the GABA agonist effect on morphine analgesia.

Animals were divided into the 4 groups: (i) a control group that received saline; (ii) a group induced morphine tolerance: received morphine once every day for 8 days (morphine tolerance group); (iii) a group induced morphine tolerance and injected muscimol ( $0.5 \mathrm{mg} /$ kg; i.p.) and (iiii) a group induced morphine tolerance and injected baclofen as GABAB agonist $(0.5 \mathrm{mg} / \mathrm{kg}$; i.p.). There were eight rats in each group. The behavioral test (Formalin test) was performed on the $8^{\text {th }}$ day in the four groups. Also, in the electrophysiological part, there were 4 groups that had a same protocol like behavioral test, but on the $8^{\text {th }}$ day instead of formalin test, the animals anesthetized and spinal cord of rats were exposed and the behaviors of WDR neurons were recorded.

\section{Drug administration}

To induce tolerance to analgesic effects, morphine hydrochloride (Temad, Iran) was chronically administered at a daily dose of $10 \mathrm{mg} / \mathrm{kg}$, i.p., from days 1 to 8 (Hill et al., 2016). To determine the effect of GABA agonists on the development of morphine tolerance, muscimol (Sigma- Aldrich, USA) was used as GABAA agonist and baclofen (Zahravi CO, Iran) 
was used as GABAB agonist, which were administrated i.p., at a dose of $0.5 \mathrm{mg} / \mathrm{kg}$ at days 1 , 3, 5 and 8, thirty minutes before injection of morphine. Then, nociceptive (Formalin test) and electrophysiological tests were performed on day $8^{\text {th }}$. All the drugs were dissolved in physiological saline.

\section{Anti-nociceptive test}

The rats were placed individually in an open Plexiglas chamber (bowl-like cage $40 \times 35 \mathrm{~cm}$ ) with a mirror angled at $45^{\circ}$ and positioned behind to allow an un-obstructed view of the paws by the observer. The animals were habituated to the observation chamber for $30 \mathrm{~min}$ prior to the experimental sessions. Formalin $(50 \mu \mathrm{l})$ was injected s.c. into the plantar surface of the rat hind paw (left or right, counterbalanced across each treatment group) using a 27-gauge needle. After injection, rats were immediately returned to the observation chamber and formalin-induced behaviors were recorded by a trained observer continuously for $60 \mathrm{~min}$. Formalin injection produced characteristic behaviors consisting of flinching and licking/biting of the injected paw. These behaviors were quantified based on pain severity. The nociceptive responses were scored every $15 \mathrm{~s}$ as follows: 0 (the injected paw is placed on the floor), 1 (the injected paw rests lightly on the floor and little or no weight is placed on it), 2 (the injected paw is elevated and not in contact with any surface), and 3 (the injected paw is licked, bitten, or shaken) (Roca-Vinardell et al., 2018). The total nociceptive score is expressed based on percentage of area under curve (AUC) over 0-5 minutes for phase I and 15-60 min for Phase II. We used one way ANOVA for evaluating of significance between data.

\section{Electrophysiology study}

Extracellular single unit recording was performed $(n=8)$ on day 8 after morphine tolerance was induced. Animals were anesthetized with $2.0-2.5 \%$ isoflurane $\left(66 \% \mathrm{~N}_{2} \mathrm{O}\right.$ and $\left.33 \% \mathrm{O}_{2}\right)$. The rat was placed in a stereotaxic frame and a laminectomy was performed on T13-L1 region of the spinal cord. A tungsten electrode (Friedrick Hear \& CO., Bowdoinham, ME, USA) was lowered into the dorsal horn while receptive fields on the ipsilateral hind paw were stimulated. Extracellular single unit activity was recorded from neurons at the depths of 500-1000 $\mu \mathrm{m}$ from the surface of the dorsal horn. Recorded signals were amplified by a data acquisition system (Science Beam CO., Tehran, Iran), and were continuously captured on a Pentium 4 computer using the e-Probe 1-42 software (Science Beam CO., Tehran, Iran). The signals were filtered using a bandwidth of 300-3000 Hz. The number of stored digital spikes for each stimulus was counted in $1 \mathrm{~ms}$ bin sizes using e-Probe spike software (Science Beam CO., Tehran, Iran) to build histogram of post-stimulus time. Besides, the responses of different fibers were separated according to their latencies (Ab-fibre 0-20 ms; Ad-fibre 20-90 ms; and C-fibre 90-300 ms). Responses that occurred after the C-fibre latency were characterized as post-discharge (300 to $800 \mathrm{~ms}$ ). Wind-up was calculated as the total number of evoked action potentials after 16 stimuli at three times. In this sense, the C-fiber threshold minus the input spike is multiplied by 16. Input spike is the number of C-fiber latencies draw out by the first electrical stimulus. The WDR neurons were identified by the depth of the microelectrode and characteristic response profiles of the neurons. After characterization of the neuron by means of natural stimuli, 16 electrical pulses 0.5 $\mathrm{Hz}, 2 \mathrm{~ms}$ wide) were applied via needles inserted into the center of the receptive field in rat paw. This provided a constant reproducible test stimulus for the experiment. Stimulation 
was applied at 3 times the threshold current for C-fiber activation and a post-stimulus histogram (PSTH) was built and displayed by e-probe software (Science beam-Iran). From the PSTH, the $C$ fiber -evoked response could be separated by latency and threshold from the $\mathrm{A} \beta, \mathrm{A} \delta$, post discharge, input spikes and wind up activity, and then quantified. Then we used one way ANOVA for evaluating of significance between data.

\section{Results}

\section{Behavioral study}

1. administration of formalin into the rats' hind paws could induce biphasic behaviors of sever pain (licking/biting/shaking ) observed between 0-5 min (first phase) and 15-60 min (second phase) as there is no responses recorded between 5 and $15 \mathrm{~min}$ (silence phase).

2. Administration of morphine (10 mg/kg daily i.p. for 8 days) to rats induces tolerance to the analgesic effects of morphine. There was no significant difference between the group that received chronic administration of morphine and the control group in both phases of the formalin test. However a single dose of morphine in non-tolerance rats provides a strong analgesic effect of the nociception stimulus in both phases of the formalin test (Figure 1) $(\mathrm{P}<0.0001)$.

3. Single dose administration of muscimol and baclofen $(0.5 \mathrm{mg} / \mathrm{kg})$ could not decrease pain induced with formalin in the controlled group (Figure 2) but repeated administration of muscimol and baclofen could significantly reduce pain in both phases of formalin test and delays to the analgesic effects of morphine tolerance development as measured by the formalin test in male tolerate rats (Figure 3) $(\mathrm{P}<0.001)$. Pretreatment with muscimol and baclofen 30 min before morphine injection in 1, 3, 5, 8 days of injection strongly influenced on morphine tolerance in 8 days and delay in the development of morphine tolerance and no tolerance developed during the experiment.

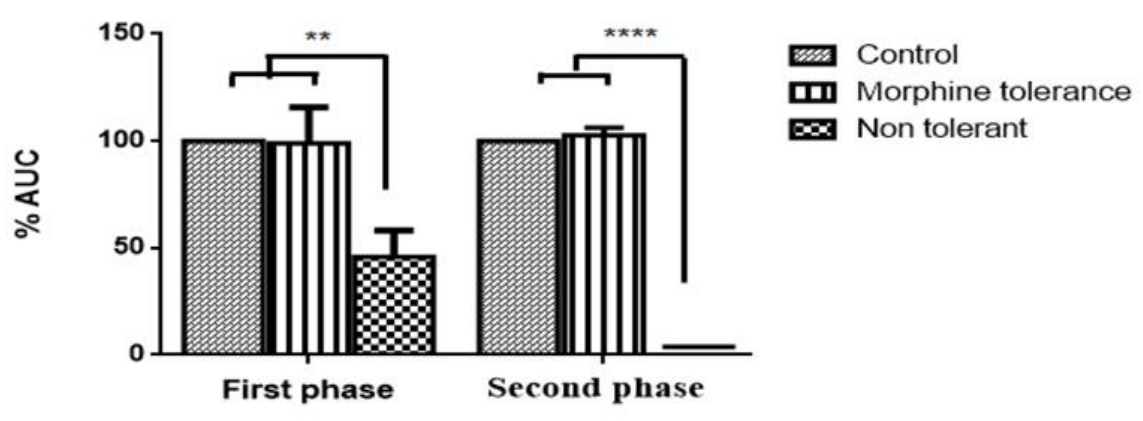

Figure 1. The effect of chronic administration of morphine on formalin pain. There is no significant differences in the antinociceptive effect of morphine between the morphine-tolerance group and the control group in both phases of the formalin test; But significant differences were found between the morphine-tolerance group and non-tolerant one $(\mathrm{n}=6$, mean $\pm \mathrm{SEM}){ }^{* *} \mathrm{p}<0.01{ }^{* * * *} \mathrm{p}<0.0001$. Statistical analyze were performed using the one-way ANOVA and Bonferroni correction tests 


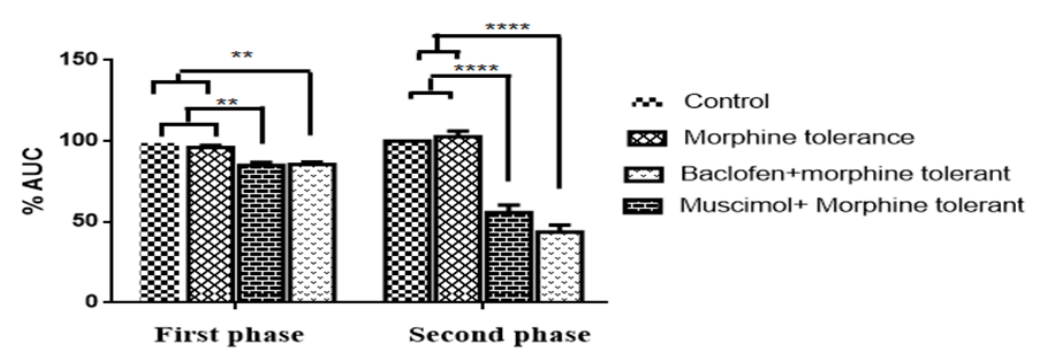

Figure 2. The effect of GABA agonists on morphine tolerance. There is significantly differences between baclofen and muscimol groups and morphine tolerance and control group. ( $\mathrm{n}=6$, mean \pm SEM) $\left.{ }^{* *} \mathrm{p}<0.01^{* * * *} \mathrm{p}<0.0001\right)$. GABA agonists reduced pain in both phases of formalin test. Compared baclofen+morphine tolerant group/morphine tolerance group and compared muscimol+morphine tolerant group/morphine tolerance group. Statistical analyze were performed using the one-way ANOVA and Bonferroni correction tests

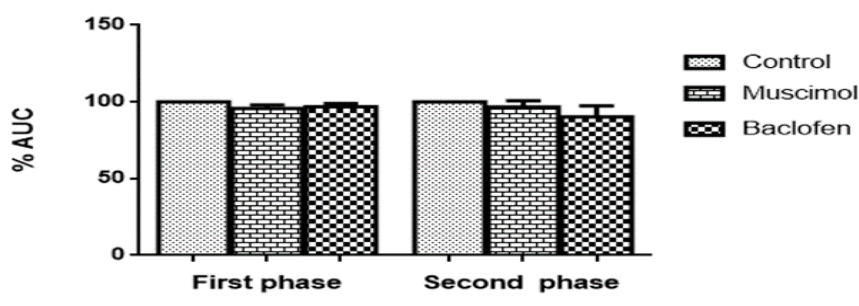

Figure 3. Antinociceptive effect of GABA agonists on non tolerant rats. Administration of GABA agonists $(0.5 \mathrm{mg} / \mathrm{kg})$ in non-tolerant rats didn't show significantly different with control group. $(\mathrm{n}=6, \mathrm{mean} \pm \mathrm{SEM})$. Compared baclofen group/control and compared muscimol group/control. Statistical analyses were performed using the one-way ANOVA and Bonferroni correction tests

\section{Electrophysiology study}

1. The Electrophysiology studies in morphine tolerate male rats in dorsal horn WDR neurons on day 8 showed that $\mathrm{A} \beta$ fiber evoked responses which were not significantly different with the controlled group. But, A $\delta$ and C-fiber evoked responses from electrical stimuli that were performed on RFs of WDR neurons in hind-paw and were higher in the morphine-treated group in comparison to the controlled group $(\mathrm{p}<0.01)$. Moreover, a comparison of WDR neurons in morphine tolerance and the control group showed significant increases in post-discharge $(\mathrm{P}<0.01)$, input spikes $(\mathrm{P}<0.05)$, and windup spikes $(\mathrm{P}<0.01)$ (Figure 4$)$.

2. The effect of administration of GABA agonists in morphine tolerate rats were analyzed. The result showed significant decreases in $A \delta$-fiber $(\mathrm{P}<0.01)$ and $\mathrm{C}$-fiber $(\mathrm{P}<0.01)$ mediated transmission to WDR neurons, as well as post-discharge $(\mathrm{P}<0.01)$, input spike $(\mathrm{P}<0.05)$ and windup spikes $(\mathrm{P}<0.01)$ in comparison to the morphine tolerance group (Figure 5). Induction of wind up, input spike and post discharge that excitability reflect WDR neurons increase in comparison to the controlled group. This result showed that the chronic administration of morphine increases activation of WDR neurons. But, GABA agonists group could significantly attenuate induction of wind up, input spike and post discharge as they couldn't return these parameters to a baseline state. Although, when we used baclofen or muscimol alone $(0.5 \mathrm{mg} / \mathrm{kg})$ with bolus injection in non-tolerate rats, there was no significant response in $\mathrm{A} \delta$ and $\mathrm{C}$-fiber evoked- 
responses in comparison to the controlled group in which the result showed that GABA agonists with this dose in non-tolerate rats don't have analgesic effect while when we used them with chronic injection of morphine, they could decrease $\mathrm{A} \delta$ and C-fiber evoked-responses, increased antinociceptive effect of morphine tolerance and prevented the development of morphine.
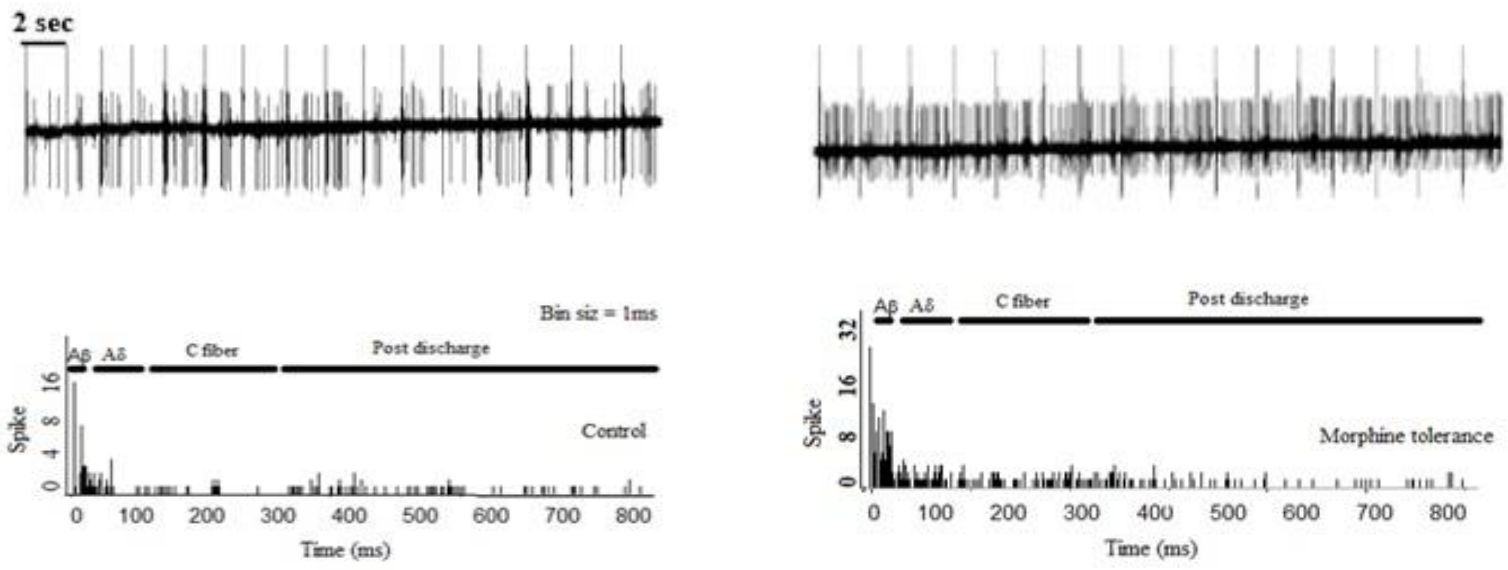

Control group

Morphine tolerance group

Figure 4. The PSTH of WDR neurons to electrical stimuli, in morphine tolerance and control groups. The responses evoked by the different fiber were quantified on the basis of latency measurements (A $\beta$-fiber, $\mathrm{A} \delta$-fiber, and $\mathrm{C}$-fiber). Results are presented as mean $\pm \mathrm{SEM}(\mathrm{n}=10)$. The $\mathrm{A} \delta$ and $\mathrm{C}$-fibre transmission onto WDR neurons were increased in morphine tolerate rats compared to control group, as well as PD, IS and Wind-up of WDR neurons $\left({ }^{*} \mathrm{P}<0.05,{ }^{* *} \mathrm{P}<0.01\right)$
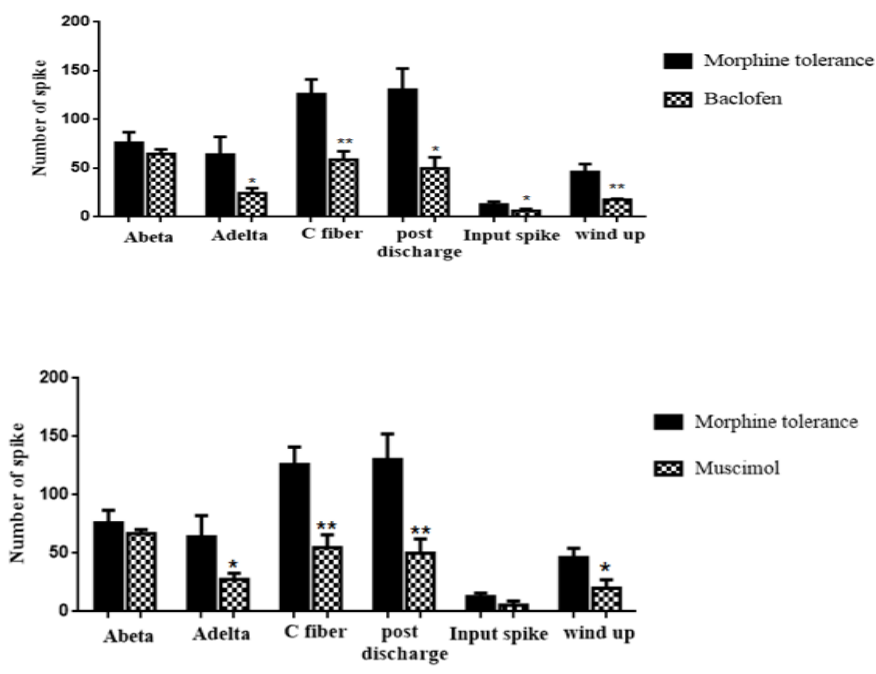

Figure 5. GABA agonists attenuate hyper activity of WDR neurons in morphine tolerate rats. GABA agonists $0.5 \mathrm{mg} / \mathrm{kg}$ inhibited $\mathrm{A} \delta$ and C-fiber mediated transmission onto WDR neurons $(\mathrm{n}=10)$ compared to morphine tolerate rats $(\mathrm{n}=10)$. GABA agonists also had a significant inhibitory effect on WDR neuronal post-discharge (PD) and inhibited wind-up, a potentiated response mediated by nociceptive $C$-fiber activity. GABA agonists had no effect to $\mathrm{A} \beta$-evoked responses since there was no difference in $\mathrm{A} \beta$ evoked responses between control and morphine tolerance group. $\left({ }^{*} \mathrm{P}<0.05,{ }^{*} \mathrm{P}<0.01\right)$ 


\section{Discussion}

In the present study, it was showed that choronic administration of morphine (i.p.: $10 \mathrm{mg} / \mathrm{kg}$ for 8 days) caused tolerance to the antinociceptive effects of morphine during the formalin test. It has been reported that intraperitoneal injection of chronic administration of morphine induces morphine tolerance in tail flick and hot plate test (Javan et al., 2003; Sepehri et al., 2010). The present data also indicated that acute administration of morphine (i.p.: $10 \mathrm{mg} / \mathrm{kg}$ ) in rats has analgesic effect during the formalin tests. Also, the result of the current study showed that i.p. administration of GABA agonists augments chronic anti-nociceptive effects of morphine and block tolerance in the formalin test. GABA agonists could suppress the induction of the wind up of $A \delta$ and C-fiber-evoked responses in single WDR spinal neurons and attenuate augmented activation of WDR neurons in morphine tolerant male rats in 8 days of morphine tolerance induction. There are other studies that confirm the present results. Using spinal cord extracellular single unit recording, another study showed that morphine tolerance increases c-fiber evoked activity and induction of LTP (Haugan et al., 2008). It has been shown that GABA has an important role in the development of morphine tolerance (Zarrindast and Mousa-Ahmadi, 1999). The interaction between opioid and GABA is a very interesting subject for study and has been studied in different models of morphine tolerance and dependence(Bannister et al., 2011; Zeng et al., 2006). There is always a controversial result with regards to GABA effects on the part of the CNS in morphine tolerance. It is established that GABA agonists can augment anti-nociceptive effect of morphine by reducing dopamine neurotransmitter in mesolimbic system (Zarrindast and Moghaddampour, 1991). Also, in this study, it was shown that GABA agonists below effective dose $(0.5 \mathrm{mg} / \mathrm{kg})$ can delay induction of morphine tolerance with impact on the spinal cord through reduction of hyper-activity of WDR neurons in morphine tolerance. Also, GABA could reduce induction of wind up in WDR neuron by attenuating C-fiber and A $\delta$-fiber activity, and also reduce the input spike; post discharge activity showed that the activity of WDR neurons decreased. In behavioral and clinical studies, it was found that chronic use of morphine causes hyperalgesia phenomenon (Hay et al., 2009). Studies on opioid tolerance and opioid-induced hyperalgesia have determined neuroplastisity alternations in the CNS. The most important site of opioid actions is the dorsal horn of the spinal cord. Interestingly, opioid induced pain and analgesia tolerance to opioid seem to share the same mechanisms with abnormal pain after peripheral nerve injury (neuropathic pains) (Mao et al., 1995). Both states are associated with reduction of anti-nociceptive effect of opioid and may be reversed by spinal GABA agonists by decreasing induction of wind up in morphine tolerance (Ji et al., 2003). Also, previous studies showed that in neuropathic pain models, WDR neuron had similar behavior with morphine tolerance model used in this experiment. This study emphasizes that (based on the result), one of the reasons for hyperalgesia development may be hyperactivity of WDR neurons which facilitates the induction of wind up in morphine tolerant rats. Also indicating the model of morphine tolerance, neuroplastic changes occurred in the spinal cord which can be similar to many models of neuropathic pain and long-term administration of morphine not only does not reduce pain, but also disrupts the pain pathway and consequently induce hyperalgesia. Also, the studies indicated that both GABA agonists could attenuate hyperalgesia in neuropathic pain and morphine tolerance in behavioral study (Patel et al., 2001; Eaton et al., 1999; Cohen and Mao, 2014). In this study, it is shown for the first time that GABA could decrease hyperactivity of WDR neurons and indicated the role of GABA agonists on the spinal level in morphine tolerance which has not been 
studied before. Both GABA agonists were used to show that activity of both receptors in the spinal cord could help to reduce morphine tolerance. For better understanding of the neuroplasticity changes in the morphine tolerance model, it is suggested that more molecular and electrophysiological studies on different types of morphine tolerance and in different sections and level of the spinal cord be conducted. In conclusion, the results of this study indicate that administration of GABA agonists is an effective way for attenuating development of morphine tolerance, and the underlying mechanism is reduction of WDR neuron hyper-responsiveness.

\section{Conclusion}

This study provides a new way for preventing the development of morphine tolerance in long term administration of morphine by GABA agonists, a general understanding on the development of morphine tolerance and the effect of chronic use of morphine and morphine along with GABA agonists on WDR neuron behaviors in spinal cord.

\section{References}

Bannister, K, Sikandar, S, Bauer, CS, Dolphin, AC, Porreca, F, Dickenson, AH. (2011). Pregabalin suppresses spinal neuronal hyperexcitability and visceral hypersensitivity in the absence of peripheral pathophysiology. Anesthesiol.: J. Am. Soc. Anesthesiolog., 115:144-152.

Belozertseva, I, Andreev, B. (2000). The effect of GABA-positive agents on the formation of morphine dependence and on the manifestations of a withdrawal syndrome. Eksp. Klin. Farmakol., 63:19-23.

Cohen, SP, Mao, J. (2014). Neuropathic pain: mechanisms and their clinical implications. $B M J: B r$. Med. J., 348:f7656

Dobashi, T, Tanabe, S, Jin, H, Nishino, T, Aoe, T. (2010). Valproate attenuates the development of morphine antinociceptive tolerance. Neurosci. Lett., 485:125-128.

Eaton, MJ, Martinez, MA, Karmally, S. (1999). A single intrathecal injection of GABA permanently reverses neuropathic pain after nerve injury. Brain Res., 835:334-339.

Haugan, F, Rygh, L, Tjølsen, A. (2008). Ketamine blocks enhancement of spinal long-term potentiation in chronic opioid treated rats. Acta Anaesthesiol. Scand., 52:681-687.

Hay, JL, White, JM, Bochner, F, Somogyi, AA, Semple, TJ, Rounsefell, B. (2009). Hyperalgesia in opioid-managed chronic pain and opioid-dependent patients. J. Pain, 10:316-322.

Hill, R, Lyndon, A, Withey, S, Roberts, J, Kershaw, Y, Maclachlan, J, Lingford-Hughes, A, Kelly, E, Bailey, C, Hickman, M. (2016). Ethanol reversal of tolerance to the respiratory depressant effects of morphine. Neuropsychopharmacology, 41:762.

Hull, L, Gabra, B, Bailey, C, Henderson, G, Dewey, W. (2013). Reversal of morphine analgesic tolerance by ethanol in the mouse. J. Pharmacol. Exp. Ther., 345:512-519.

Javan, M, Motamedi, F, Ahmadiani, A, Masoudnia, F. (2003). Possible relevance of tolerance to analgesic effect of morphine due to chronic inflammatory pain and the role of lumbar spinal cord in this interaction. Physiol. Pharmacol., 7:3-11. 
Ji, RR, Kohno, T, Moore, KA, Woolf, CJ. (2003). Central sensitization and LTP: do pain and memory share similar mechanisms? Trends neurosci., 26:696-705.

Lilius, T, Kangas, E, Niemi, M, Rauhala, P, Kalso, E. (2017). Ketamine and norketamine attenuate oxycodone tolerance markedly less than that of morphine: from behaviour to drug availability. British Journal of Anaesthesia., 120:818-826

Lueptow, LM, Fakira, AK, Bobeck, EN. (2018). The contribution of the descending pain modulatory pathway in opioid tolerance. Front. Neurosci, 12:886.

Mansouri, MT, Khodayar, MJ, Tabatabaee, A, Ghorbanzadeh, B, Naghizadeh, B. (2015). Modulation of morphine antinociceptive tolerance and physical dependence by co-administration of simvastatin. Pharmacol. Biochem. Behavi., 137:38-43.

Mao, J, Price, DD, Mayer, DJ. (1995). Mechanisms of hyperalgesian and morphine tolerance: a current view of their possible interactions. Pain, 62:259-274.

Mehrabadi, S, Karimiyan, SM. (2018). Morphine Tolerance Effects on Neurotransmitters and Related Receptors: Definition, Overview and Update. J. Pharmaceut. Res. Internat, 23:1-11.

Patel, S, Naeem, S, Kesingland, A, Froestl, W, Capogna, M, Urban, L, Fox, A. (2001). The effects of GABAB agonists and gabapentin on mechanical hyperalgesia in models of neuropathic and inflammatory pain in the rat. Pain, 90:217-226.

Roca-Vinardell, A, Berrocoso, E, Llorca-Torralba, M, García-Partida, J, Gibert-Rahola, J, Mico, J. (2018). Involvement of 5-HT1A/1B receptors in the antinociceptive effect of paracetamol in the rat formalin test. Neurobiol. Pain, 3:15-21.

Sepehri, G, Sheibani, V, Azarang, A, Shamsizadeh, A, Afarinesh, MR, Azizollahi, S, Sepehri, E. (2010). The intracerebroventricular (ICV) administration of W-7, a calmodulin inhibitor, attenuates the development of morphine tolerance in rats. Pak. J. Pharm. Sci., 23:170-174.

Vorma, H, Katila, H. (2011). Effect of valproate on benzodiazepine withdrawal severity in opioiddependent subjects: a pilot study. Heroin. Addict. Relat. Clin. Probl., 13:15-20.

Zarrindast, MR, Mousa-Ahmadi, E. (1999). Effects of GABAergic system on naloxone-induced jumping in morphine-dependent mice. Eur. J. Pharmacol., 381:129-133.

Zarrindast, M, Moghaddampour, E. (1991). Influences of dopamine agonists and antagonists of baclofen antinociception in mice. Arch. Int. pharmacodyn. Ther., 309:42-50.

Zeng, J, Thomson, LM, Aicher, SA, Terman, GW. (2006). Primary afferent NMDA receptors increase dorsal horn excitation and mediate opiate tolerance in neonatal rats. J. Neurosci, 26:12033-12042.

How to cite this article: Shima Mehrabadi, Homa Manaheji. Effect of Sub-effective dose of GABA Agonists on Attenuation of Morphine Tolerance in Rats: Behavioral and Electrophysiological Studies. International Journal of Advanced Biological and Biomedical Research, 2019, 7(4), 326-334. Link: http://www.ijabbr.com/article 35425.html 